Degenerate quantum gases manipulation on AtomChips

This article has been downloaded from IOPscience. Please scroll down to see the full text article. 2012 Phys. Scr. 2012014002

(http://iopscience.iop.org/1402-4896/2012/T149/014002)

View the table of contents for this issue, or go to the journal homepage for more

Download details:

IP Address: 130.236.83.211

The article was downloaded on 27/02/2013 at 14:39

Please note that terms and conditions apply. 


\title{
Degenerate quantum gases manipulation on AtomChips
}

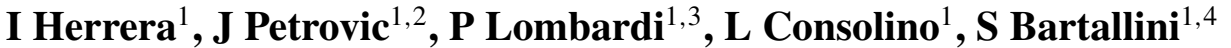 \\ and F S Cataliotti ${ }^{1,5}$ \\ ${ }^{1}$ European Laboratory for Nonlinear Spectroscopy (LENS), Via Nello Carrara 1, 50019 Sesto F.no (FI), \\ Italy \\ ${ }^{2}$ Vinča Institute of Nuclear Sciences, PO Box 522, 11001 Belgrade, Serbia \\ ${ }^{3}$ Dipartimento di Fisica e Astronomia Università di Firenze via Sansone 1, 50019 Sesto F.no (FI), Italy \\ ${ }^{4}$ Istituto Nazionale di Ottica-CNR, Largo E Fermi 6, 50125 Firenze, Italy \\ ${ }^{5}$ Dipartimento di Energetica 'Sergio Stecco' Università di Firenze via S Marta 3, 50139 Firenze, Italy
}

Received 15 September 2011

Accepted for publication 19 October 2011

Published 27 April 2012

Online at stacks.iop.org/PhysScr/T149/014002

\begin{abstract}
The integration of cold atomic sources and electromagnetic field sources in the same device is a major frontier for both fundamental science and advanced technology. In this paper, we discuss the realization of the Bose-Einstein condensate (BEC) on an AtomChip and describe such an experimental configuration realized at LENS. We also concentrate on the realization of a particular integrated matter wave interferometer where coherent coupling between the BECs in different Zeeman states is used to produce a fringe time-population signal at the output. Finally, we discuss the prospects for application of such a device as a multi-path interferometer.
\end{abstract}

PACS numbers: 67.85.Fg, 37.25.+k

(Some figures may appear in colour only in the online journal)

\section{Introduction}

Cold atomic systems and degenerate quantum gases are unique tools for the modeling of quantum systems [1] and for precision measurements of atom characteristics beyond the classical limit [2]. In particular, the realization of a Bose-Einstein condensate (BEC) [3] as a coherent state of matter [4] has advanced matter-wave interferometry considerably. Long coherence times and the localization in phase space of cold-atom clouds and, in particular, of BECs enable high-precision interferometric measurements of the internal properties of atoms, many-body effects and gravity [5]. However, their applications outside the laboratory depend critically on the compactness and robustness of interferometric setups and hence on the simplification and downsizing of bulky cold-atom setups. An important invention in this direction is the realization of a BEC in microscopic magnetic traps based on micro-electronics technology, yielding the so-called AtomChip [6,7]. Thanks to components' miniaturization, one can reach extremely large magnetic field gradients (of the order of $\mathrm{Tcm}^{-1}$ ) with a few amperes current, similar to those flowing in ordinary electronic circuits. Moreover, AtomChips can be integrated with wires [6] and photonic components [9] that can generate control potentials for atom manipulation. Experiments based on AtomChips have allowed the demonstration of trapped atom interferometers [8], Michelson interferometers [10] and nonlinear interferometers with sensitivity beyond the standard quantum limit [2].

In this paper we present a scheme and experimental demonstration of a time-domain interferometer fully integrated on an AtomChip. The interferometer is based on the state-dependent evolution of atoms in a magnetic field. Section 2 describes the principle of operation of AtomChips and provides details of the realization of a BEC on the AtomChip at LENS. Section 3 introduces the interferometric scheme and a two-path interferometer on this chip. Finally, in section 4 we review the advantages and disadvantages of the proposed system and briefly discuss its possible generalization to a multi-path interferometer. 

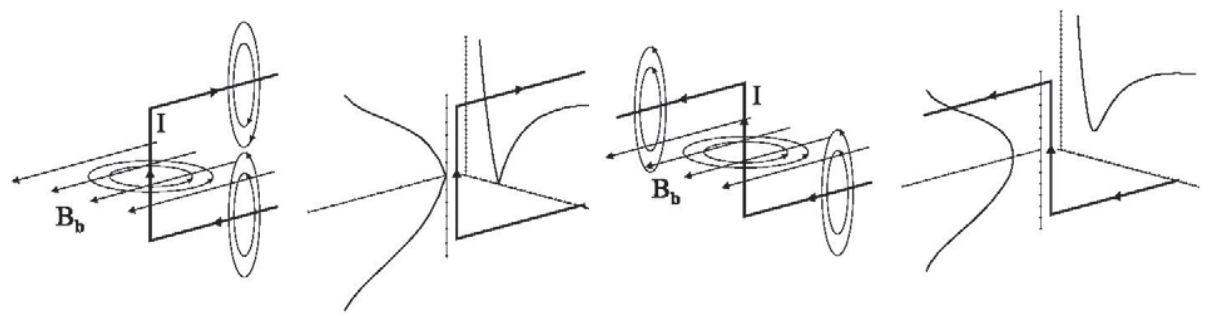

Figure 1. Microtrap configurations. Left: the quadruple U-wire trap. Right: the Ioffe-Pritchard Z-wire trap.

\section{Bose-Einstein condensation on an AtomChip}

The standard experimental procedure for the creation of a BEC in a dilute atomic gas starts with laser cooling of an atomic vapor in ultra-high vacuum conditions. The first step, performed in a magneto-optical trap [11], takes the atoms from a phase space density of $10^{-20}$ at room temperature to $10^{-5}$ below $100 \mu \mathrm{K}$, when the laser cooling stops mainly due to the spontaneous light scattering from the atoms [12-14]. For this reason, another cooling step has to be performed in a non-dissipative trap created either by a magnetic field or by a very far-off resonance laser beam. Conventional magnetic traps are realized with a few centimeters size coils carrying more than $100 \mathrm{~A}$ of current. In this way traps with $10-100 \mathrm{~Hz}$ oscillation frequencies are realized. In non-dissipative traps, cooling is achieved by removing the high-energy tail of the atomic distribution and by letting the atoms thermalize via binary collisions. Removal of atoms is realized either by reducing the trap depth in optical traps or by radiofrequency (RF)-induced transitions to untrapped Zeeman sublevels in magnetic traps. The evaporation time in conventional traps is of the order of $1 \mathrm{~min}$, which sets very stringent requirements on the vacuum system. In particular, the required vacuum is hardly compatible with the loading of atoms in a magnetic optical trap, forcing one to use a double-magnetic optical trap system where loading and trapping are separated. In contrast, a miniaturized magnetic trap such as that realized on an AtomChip offers much higher trap frequencies, thus significantly reducing the experimental complexity.

AtomChips are based on the possibility of creating a trapping field close to a current-carrying wire by compensating for the field generated by the wire $B=\frac{\mu_{0}}{\pi} \frac{I}{r}$ by a constant magnetic field (bias). The presence of a bias field creates a zero of the magnetic field on an axis parallel to the wire direction at a height $z_{0}$. Near this axis the field can be approximated with a two-dimensional (2D) quadrupole, thus creating a linear guide for spin aligned atoms. By controlling the chip wire current and the bias field, the trap center can be brought in close proximity to the wire, where a high magnetic field gradient ensures tight trapping. For example, with a current of just $0.4 \mathrm{~A}$ in the wire and a constant field of $35 \mathrm{G}$, it is possible to create a waveguide with a confining frequency of $10 \mathrm{kHz}$ for ${ }^{87} \mathrm{Rb}$, two orders of magnitude larger than that of the conventional magnetic traps.

The wire guide represents a building brick for magnetic microtraps. Indeed, by bending the wire in a $U$ shape it is possible to create a $3 \mathrm{D}$ quadrupole since the magnetic fields created by the arms of the $\mathrm{U}$ cancel in the middle, see figure 1 (left). However, this trap configuration has high atomic losses due to Majorana spin-flips [17]. In order to avoid these losses,

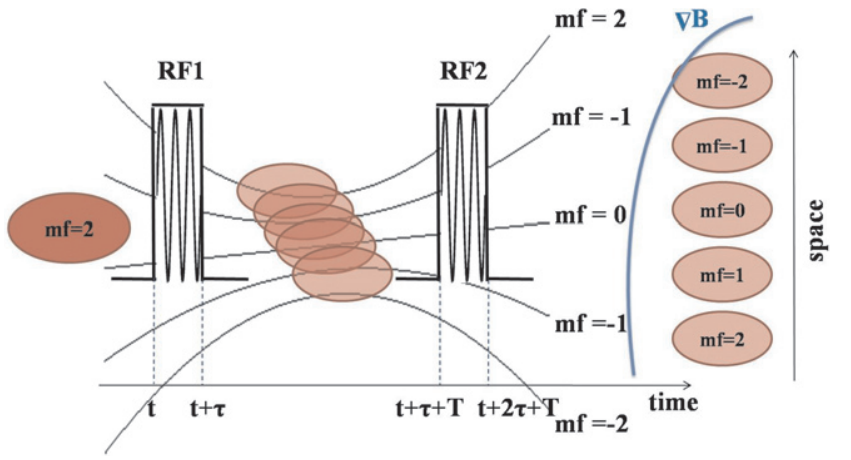

Figure 2. Scheme of the interferometer. Two RF pulses act as Rabi pulses, the trap magnetic field provides the energy difference between the Zeeman states and the chip wire magnetic field gradient separates these states in space for imaging.

it is better to create a harmonic trap with a field minimum different from zero as can be done by bending the wire in a $\mathrm{Z}$ shape, see figure 1 (right).

In our experiment, an AtomChip [15, 16] carrying micrometer-wide guiding wires was mounted on a holder (Shapal ceramics) with an embedded Z- and two U-wires. It was mounted facing downwards and was closed in a high-vacuum cell. Golden chip coating was used to construct a reflection magneto-optical trap for ${ }^{87} \mathrm{Rb}$ atoms. The atoms were further cooled to $10 \mu \mathrm{K}$ by the molasses and then pumped into the $\left|F=2, m_{\mathrm{F}}=2\right\rangle$ state before two-stage loading into the chip magnetic trap. First, an ancillary magnetic trap was generated at $1.2 \mathrm{~mm}$ from the chip by a $25 \mathrm{~A}$-guiding Z-wire in the chip holder. This trap was subsequently compressed and lowered while the chip trap was ramped up. The final magnetic trap was formed by a 1.7 A-guiding chip Z-wire and had the frequencies $\left(v_{\perp}, v_{z}\right)=$ $(950 \mathrm{~Hz}, 46 \mathrm{~Hz})$. The atoms were evaporated to quantum degeneracy by a ramped RF field supplied by an RF generator (Agilent 33250A) and a U-wire in the chip holder. The BEC had $3 \times 10^{4}$ atoms and the critical temperature of $0.5 \mu \mathrm{K}$ and was $200 \mu \mathrm{m}$ from the chip surface. The whole procedure takes $8.3 \mathrm{~s}$. We wait another $15 \mathrm{~s}$ before repeating the cycle to restore the vacuum and reduce heating of the chip assembly. The atoms were detected by a $7.5 \times$-magnifying absorption imaging system and a high-resolution camera (SIS1-s285, Theta-System). The error of the population measurement was $5 \%$.

\section{Manipulation of atomic internal states}

The main components of the interferometer proposed here are shown in figure 2 . The initial state was the $\left\langle F=2, m_{\mathrm{F}}=2\right\rangle$ 

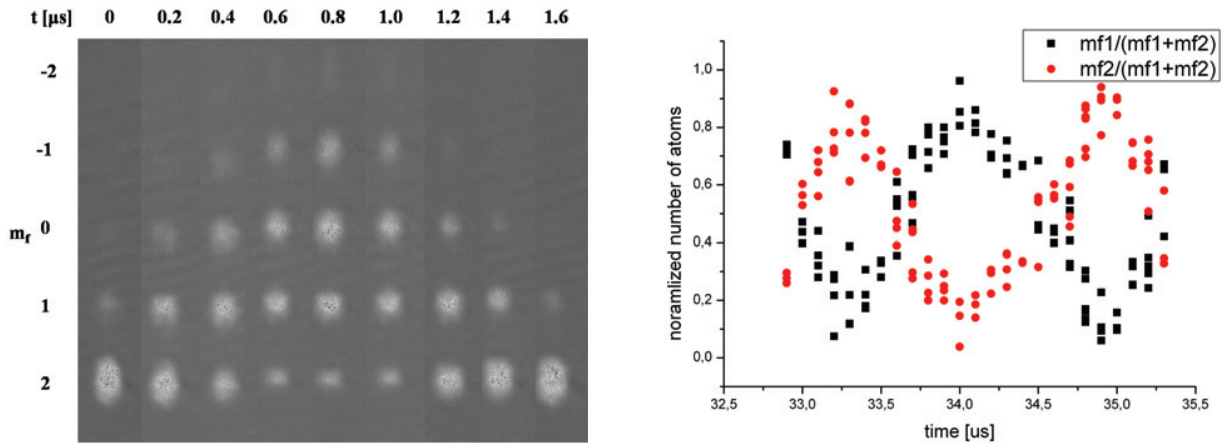

Figure 3. A two-path interferometer realized by $\pi / 2$ RF Rabi pulses supplied by an antenna on chip. Left: a sequence of absorption imaging frames taken at different time delays. Right: populations of $m_{\mathrm{F}}=2$ and $m_{\mathrm{F}}=1$ states versus the time delay as obtained by integration of the absorption images.

condensate described above. Coherent transfer of atoms to other Zeeman sublevels is realized by the application of a resonant RF pulse and the interferometer is closed by the application of another identical RF pulse after a controllable time delay. In the trapping magnetic field $\mathbf{B}$, Zeeman states experience different potentials given by $V=m_{\mathrm{F}} g_{\mathrm{F}} \mu_{0}|B|$, where $m_{\mathrm{F}}$ and $g_{\mathrm{F}}$ are, respectively, spin and Landè numbers and $\mu_{0}$ is the Bohr magneton. Therefore, their relative phases develop with frequencies equal to multiples of the energy separation between any two adjacent levels $\omega=g_{\mathrm{F}} \mu_{0}|\mathbf{B}| / \hbar$. Thus, accumulated phase differences between the states determine the populations of each state upon remixing by the second RF pulses. In order to measure the population of each state, first they are separated in space by the application of the Stern-Gerlach method followed by free-fall expansion, and then they are imaged by absorption imaging.

The interferometric signal is the normalized state population as a function of the time delay between the pulses. The sharpness of the fringes and hence the sensitivity of the interferometer are determined by the number of states present between the two pulses. Since the frequency separations between the Zeeman states are multiples of the separation between two adjacent states, the larger the number of participating states, the more harmonics will be present in the output signal. Here we concentrate on a simple interferometer formed by the two trapped states, the low-field seeking $m_{\mathrm{F}}=2$ and $m_{\mathrm{F}}=1$. A more sensitive interferometer formed by using a higher number of states will be described elsewhere.

The RF pulses used in the manipulation of internal states of atoms were generated by using an Agilent 33250A U-wire pair. Their frequency was set to $700 \mathrm{KHz}$ to match the separation of the Zeeman sublevels in the magnetic trap. The phase of each pulse was locked to its trigger. The Zeeman states were separated for imaging by switching off the bias field of the chip trap and letting the atoms move in the field gradient of the chip Z-wire. The measurements were repeated at least three times at each point. For each measurement a new $\mathrm{BEC}$ was created.

Figure 3 shows the oscillation in state populations obtained by the application of $\pi / 2$ Rabi pulses. Such a Rabi pulse places $41 \%$ of the atoms in each of the trapped states and the rest in the untrapped states, thereby creating a nearly two-path interferometer. Integration of the absorption images gives the interferometric signals shown in figure 3.
The oscillation period is dictated by the energy separation between the adjacent Zeeman states at the minimum of the magnetic trap, here roughly $1 \mathrm{G}$. The signals are normalized to the sum of populations in the $m_{\mathrm{F}}=1$ and $m_{\mathrm{F}}=2$ states. The loss to the other states is, however, not negligible and should be taken into account when calculating the interferometer visibility. Another process that results in a reduction of the visibility is dephasing due to the relative movement of the condensates in their respective magnetic traps. We have measured the corresponding visibility decay time of about $200 \mu \mathrm{s}$.

The proposed interferometer can be applied as a sensor of magnetic or light fields whose interaction with atoms is state dependent. The simplest example is the response of the interferometer to a magnetic field, which is observed as a change in the fringe period.

\section{Conclusions and outlook}

In conclusion, we have constructed an atom interferometer on chip in which the coherent mixing of Zeeman states generates a fringe pattern in the state-population signals at the output. Coherent splitting of the initial condensate and remixing of the Zeeman states are achieved by two RF Rabi pulses delayed with respect to each other. The RF pulses are provided by an antenna in the chip holder, which makes the whole setup compact and eliminates the need for interferometer alignment. Since its paths are not spatially resolved, the interferometer can be used in measurements involving external signals that have different impact on different Zeeman states, such as light pulses or magnetic fields. The external signal length is limited by dephasing due to the relative movement of the condensates in different Zeeman states. A disadvantage of this two-path interferometer-the loss of atoms into untrapped states - can be turned into its advantage by including these states as additional paths. Such a multi-path interferometer has an improved sensitivity and can be achieved by using Rabi pulses with larger areas.

\section{Acknowledgments}

We thank M Schramböck (Atominstitut, TU-Wien) at the ZMNS who realized the AtomChip we used. The chip was supplied through the CHIMONO Collaboration. Funding for this work was obtained from the Future 
and Emerging Technologies (FET) programme within the Seventh Framework Programme for Research of the European Commission, under FET-Open grant MALICIA (265522), and from the Italian Ministry of Research (Project HYTEQ).

\section{References}

[1] Jaksch D, Bruder C, Cirac J I, Gardiner C W and Zoller P 1998 Phys. Rev. Lett. 813108

[2] Gross C, Zibold T, Nicklas E, Estève J and Oberthaler M K 2010 Nature 4641165

[3] Inguscio M, Stringari S and Wieman C E 1998 Proc. CXL Int. School of Physics 'E. Fermi' on 'Bose-Einstein Condensation in Atomic Gases' (Amsterdam: IOS)

[4] Andrews M R, Townsend C G, Miesner H J, Durfee D S, Kurn D M and Ketterle W 1997 Science 275637

[5] Cronin A D, Schmiedmayer J and Pritchard D 2009 Rev. Mod. Phys. 811051

[6] Hänsel W, Reichel J, Hommelhoff P and Hänsch T W 2001 Phys. Rev. Lett. 86608
[7] Folman R, Krüger R, Schmiedmayer J, Denschlag J and Henkel C 2002 Adv. At. Mol. Opt. Phys. 48263

[8] Hänsel W, Reichel J, Hommelhoff P and Hänsch T W 2001 Phys. Rev. A 64063607

[9] Kohnen M, Succo M, Petrov P G, Nyman R A, Trupke M and Hinds E A 2011 Nature Photon. 535

[10] Wang Y-J, Anderson D Z, Bright V M, Cornell E A, Diot Q, Kishimoto T, Prentiss M, Saravanan R A, Segal S R and Wu S 2005 Phys. Rev. Lett. 94090405

[11] Raab E L, Prentiss M, Cable A, Chu S and Pritchard D E 1987 Phys. Rev. Lett. 592631

[12] Chu S 1998 Rev. Mod. Phys. 70685

[13] Cohen-Tannoudji C N 1998 Rev. Mod. Phys. 70707

[14] Phillips W D 1998 Rev. Mod. Phys. 70721

[15] Groth S, Krüger P, Wildermuth S, Folman R, Fernholz T, Mahalu D, Bar-Ioseph I and Schmiedmayer J 2004 Appl. Phys. Lett. 852980

[16] Trinker M, Groth S, Haslinger S, Manz S, Betz T, Bar-Ioseph I, Schneider S, Schumm T and Schmiedmayer J 2008 Appl. Phys. Lett. 92254102

[17] Majorana E 1932 Nuovo Cimento 943 